\title{
Dispersion-engineered nanophotonic devices based on subwavelength metamaterial waveguides
}

\author{
David González-Andrade ${ }^{1, *}$, Antonio Dias², José Manuel Luque-González ${ }^{3}$, J. Gonzalo Wangüemert-Pérez ${ }^{3}$, Alejandro Ortega- \\ Moñux $^{3}$, Robert Halir ${ }^{3,4}$, Íñigo Molina-Fernández ${ }^{3,4}$, Pavel Cheben ${ }^{5}$, Aitor V. Velasco ${ }^{1}$ \\ ${ }^{l}$ Instituto de Óptica Daza de Valdés, Consejo Superior de Investigaciones Científicas (CSIC), Madrid 28006, Spain \\ ${ }^{2}$ Alcyon Photonics S.L., Madrid 28004, Spain \\ ${ }^{3}$ Universidad de Málaga, Departamento de Ingeniería de Comunicaciones, ETSI Telecomunicación, Málaga 29071, Spain \\ ${ }^{4}$ Bionand Center for Nanomedicine and Biotechnology, Parque Tecnológico de Andalucía, Málaga 29590, Spain \\ ${ }^{5}$ National Research Council Canada, 1200 Montreal Road, Bldg. M50, Ottawa K1A OR5, Canada \\ *david.gonzalez@csic.es
}

\begin{abstract}
Subwavelength metamaterial waveguides are becoming an essential tool for the design of high-performance nanophotonic devices implemented on the silicon-on-insulator platform. Here we focus on dispersion engineering and present our latest advances in this field, including mode multiplexers/demultiplexers and passive phase shifters with ultra-broad bandwidths.
\end{abstract}

Keywords-subwavelength gratings, metamaterials, dispersion engineering, ultra-broadband, mode converter and multiplexer, phase shifter, silicon-on-insulator, silicon photonics

\section{INTRODUCTION}

In recent years, the silicon-on-insulator (SOI) platform has become ubiquitous for the implementation of photonic integrated circuits (PICs) operating at the near-infrared band [1]. This material platform leverages the maturity of complementary metal-oxide-semiconductor (CMOS) fabrication processes reached by the microelectronics industry, resulting in an affordable mass production. In addition to the cost effectiveness, the high refractive index contrast of the SOI platform enables to increase the number of photonic devices integrated on a single chip and, at the same time, the development of more complex systems. However, this large difference between the refractive index of the silicon waveguide core and the silicon dioxide $\left(\mathrm{SiO}_{2}\right)$ layers (i.e. $\Delta n \approx 2$ ) also poses important limitations, namely a strong modal dispersion and a high birefringence between the transverse electric (TE) and transverse magnetic (TM) polarizations. While the former results in photonic devices with narrow operating bandwidth, the latter typically limits their operation to a single polarization state of light waves, both limiting the performance of $\mathrm{Si}$ photonic devices. To overcome these constraints, subwavelength grating (SWG) metamaterial waveguides have been postulated as an essential tool for the design of high-performance nanophotonic devices implemented on the SOI platform [2].

\section{SubWAVELENGTH METAMATERIALS}

Figure 1(a) shows the schematic of an SWG metamaterial waveguide, which is composed of different dielectric materials that alternate with a period $(\Lambda)$ smaller than half the wavelength of the light propagating through the structure, thus preventing both diffraction and Bragg reflection. The key concept is that the silicon SWG waveguide is an inhomogeneous isotropic medium that acts as a homogeneous anisotropic medium [3] as shown in Fig. 1(b), specifically as a uniaxial crystal [4] with a refractive index tensor $\boldsymbol{n}_{\boldsymbol{e q}}=\operatorname{diag}\left[n_{x x}, n_{x x}, n_{z z}\right]$. In this way, designers can a) SWG waveguide

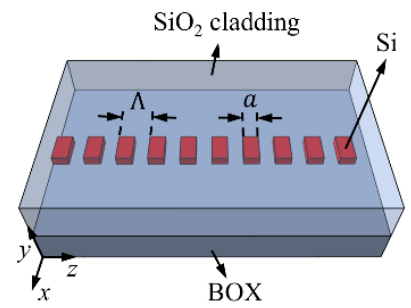

b) Equivalent metamaterial waveguide

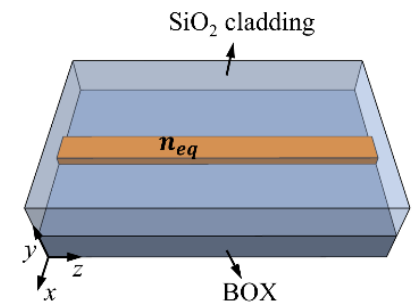

Fig. 1. (a) Schematic of a silicon SWG waveguide surrounded by a buried oxide layer and a $\mathrm{SiO}_{2}$ upper cladding. (b) Schematic of the equivalent anisotropic metamaterial defined with the refractive index tensor $\boldsymbol{n}_{\boldsymbol{e q}}$.

leverage the extended design space in order to tailor the optical properties of the equivalent anisotropic metamaterial and develop novel photonic devices with enhanced performance. Depending on the optical property exploited for the design, applications of SWG metamaterial waveguides can be divided into three groups: local refractive index, anisotropy and dispersion engineering [5-7].

In this work, we focus on engineering the dispersion properties of SWG metamaterial waveguides to achieve ultra-broadband photonic devices and present our latest advances in this field, including experimental results of a mode multiplexer-demultiplexer link and a novel $90^{\circ}$ passive phase shifter (PS).

\section{MODE CONVERTER AND MULTIPLEXER}

Our mode converter and multiplexer/demultiplexer (mux/demux) is based on a multimode interference (MMI) coupler, a $90^{\circ}$ phase shifter (PS) and a symmetric Y-junction. Theoretically, both the $90^{\circ} \mathrm{PS}$ and the symmetric $\mathrm{Y}$-junction present low losses over a broad bandwidth; however, the use of a conventional MMI coupler would intrinsically limit the performance of the complete mode mux/demux [8]. On this account, we propose to replace the conventional MMI with an SWG MMI (see Fig. 2(a)). By judiciously choosing the duty cycle $(\mathrm{DC}=a / \Lambda)$ and the period of the SWG MMI, anisotropy and dispersion can be engineered to mitigate the wavelength dependence of the MMI's beat length, thus achieving a broader operation bandwidth compared to its conventional counterpart [9].

On the other hand, the limited the limited resolution of the fabrication process typically generates a tip of nonnegligible dimensions between the arms of the symmetric Yjunction, which results in the excitation of undesired higherorder modes supported by the multimode waveguide of a mux-demux link. In order to avoid this problem, two linear 


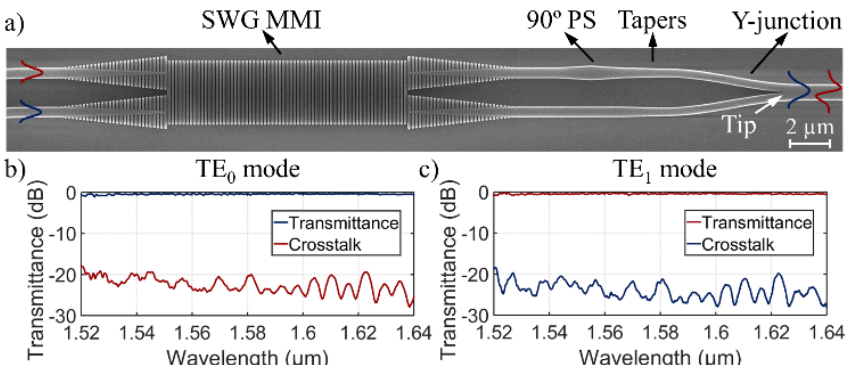

Fig. 2. (a) SEM image of the fabricated mode converter and mux/demux Measured transmittance of the complete mux-demux link when (b) the $\mathrm{TE}_{0}$ and (c) the $T E_{1}$ modes are generated in the multimode waveguide.

tapers between the $90^{\circ}$ PS and the symmetric Y-junction were included in the fabricated device to reduce the arm widths of the Y-junction and thereby de width of the multimode waveguide to support only $\mathrm{TE}_{0}$ and $\mathrm{TE}_{1}$ modes within the $1.495-1.64 \mu \mathrm{m}$ wavelength range.

A complete mux-demux link, i.e. two mode converters and mux/demux in back-to-back configuration, was fabricated using a 220 -nm-thick SOI platform with a $2-\mu \mathrm{m}-$ thick buried oxide (BOX) layer and a 2.2- $\mu$ m-thick $\mathrm{SiO}_{2}$ cladding. Experimental results of the optimized mux-demux link are shown in Figs. 2(b) and 2(c) for $\mathrm{TE}_{0}$ and $\mathrm{TE}_{1}$ modes, respectively. Insertion loss less than $1.1 \mathrm{~dB}$ and crosstalk better than $-18 \mathrm{~dB}$ for both $\mathrm{TE}_{0}$ and $\mathrm{TE}_{1}$ modes are achieved for a broad $120 \mathrm{~nm}$ bandwidth $(1.52-1.64 \mu \mathrm{m})$.

\section{NANOPHOTONIC PHASE SHIFTER}

Optical PSs are fundamental components in PICs to induce a specific phase shift between different signals. Here we propose a passive $90^{\circ}$ PS based on two parallel SWG metamaterial waveguides of dissimilar widths but with the same length, period and DC [10]. The widths of both waveguides must be wide enough to hold paraxiality condition and, at the same time, to improve tolerances to width deviations. To this end, SWG tapers are incorporated to perform an adiabatic transition between the non-periodic interconnection waveguides and the corresponding SWG waveguide (see Fig. 3(a)). The wavelength dependence of the phase shift error (PSE), defined as the deviation from the target $90^{\circ}$ phase shift, can also be mitigated by properly choosing the values of $\Lambda$ and DC of both SWG waveguides.

The device was fabricated on the same SOI platform as the mode converter and mux/demux. To experimentally characterize the fabricated PSs, we used Mach-Zehnder interferometer (MZI) consisting of two SWG MMIs and 14 PSs connected in series. We developed a circuit model to simulate the MZI and estimate the phase error introduced by fabrication deviations [10]. These errors were subtracted from the measured phase shift response and the resulting experimental results are shown in Fig. 3(b). A phase slope as small as $16 \%$ m for our proposed SWG PS, whereas a conventional PS based on two trapezoidal tapers in back-toback yields a phase slope of $64 \% / \mu \mathrm{m}$.
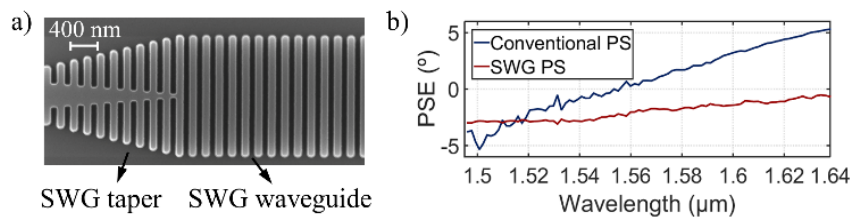

Fig. 3. (a) SEM image of the upper arm of the proposed SWG PS. (b) Measured PSE of the SWG PS (red curve) compared to the PSE of a conventional PS (blue curve).

\section{CONCLUSIONS}

The versatility offered by SWG metamaterial waveguides opens up a wide range of possibilities for the design of highperformance integrated optical devices. In this work, we have focused on dispersion engineering, demonstrating a mode converter and mux/demux and a passive PS with measured bandwidths in excess of $145 \mathrm{~nm}$. We believe that both devices can find potential applications in future cuttingedge photonics applications. In particular, the mode converter and mux/demux is a promising candidate for the implementation of broadband integrated MDM transceivers, whereas the SWG PS has remarkable prospects in coherent communications, polarimetry, quantum photonics, sensing and high-performance MDM transceivers.

\section{ACKNOWLEDGMENT}

This work has been funded in part by the Spanish Ministry of Science, Innovation and Universities (MICINN) under grants CDTI SNEO-20181232 (Alcyon Photonics S.L.), TEC2015-71127-C2-1-R (FPI scholarship BES-2016077798), TEC2016-80718-R, and RTI2018-097957-B-C33; the Spanish Ministry of Education, Culture and Sport (MECD) (FPU16/06762); and the Community of Madrid FEDER funds (S2018/NMT-4326). This project has received funding from the Horizon 2020 research and innovation program under the Marie Sklodowska-Curie grant No. 734331 .

\section{REFERENCES}

[1] W. N. Ye and Y. Xiong, "Review of silicon photonics: history and recent advances,” J. Mod. Opt., vol. 60, pp. 1299-1320, October 2013.

[2] R. Halir, A. Ortega-Moñux, D. Benedikovic, G. Z. Mashanovich, J. G. Wangüemert-Pérez, J. H. Schmid, Í. Molina-Fernández, and P. Cheben, "Subwavelength-grating metamaterial structures for silicon photonic devices," Proc. IEEE, vol. 106, pp. 2144-2157, August 2018

[3] P. Cheben, R. Halir, J. H. Schmid, H. A. Atwater, and D. Smith, "Subwavelength integrated photonics," Nature, vol. 560, pp. 565-572, August 2018.

[4] C. Gu and P. Yeh, "Form birefringence dispersion in periodic layered media", Opt. Lett., vol. 21, pp. 504-506, April 1996.

[5] P. Cheben, D.-X. Xu, S. Janz, and A. Densmore, "Subwavelength waveguide grating for mode conversion and light coupling in integrated optics,” Opt. Express, vol. 14, pp. 4695-4702, May 2006.

[6] J. M. Luque-González, A. Herrero-Bermello, A. Ortega-Moñux, Í. Molina-Fernández, A. V. Velasco, P. Cheben, J. H. Schmid, S. Wang, and R. Halir, "Tilted subwavelength gratings: controlling anisotropy in metamaterial nanophotonic waveguides," Opt. Lett., vol. 43, pp. 4691-4694, September 2018.

[7] R. Halir, A. Maese-Novo, A. Ortega-Moñux, I. Molina-Fernández, J. G. Wangüemert-Pérez, P. Cheben, D.-X. Xu, J. H. Schmid, and S. Janz, "Colorless directional coupler with dispersion engineered subwavelength structure," Opt. Express, vol. 20, pp. 13470-13477, May 2012.

[8] D. González-Andrade, J. G. Wangüemert-Pérez, A. V. Velasco, A. Ortega-Moñux, A. Herrero-Bermello, I. Molina-Fernández, R. Halir, and P. Cheben, "Ultra-broadband mode converter and multiplexer based on sub-wavelength structures," IEEE Photonics J., vol. 10, p. 2201010 , March 2018.

[9] R. Halir, P. Cheben, J. M. Luque-González, J. D. SarmientoMerenguel, J. H. Schmid, G. Wangüemert-Pérez, D.-X. Xu, S. Wang, A. Ortega-Moñux, and Í. Molina-Fernández, "Ultra-broadband nanophotonic beamsplitter using an anisotropic sub-wavelength metamaterial," Laser Photon. Rev., vol. 10, pp. 1039-1046, November 2016.

[10] D. González-Andrade, J. M. Luque-González, J. G. WangüemertPérez, A. Ortega-Moñux, P. Cheben, Í. Molina-Fernández, and A. V. Velasco, "Ultra-broadband nanophotonic phase shifter based on subwavelength metamaterial waveguides," Photon. Res., vol. 8, pp. 359-367, February 2020. 